ISSN 0103-8478

\title{
Painéis de partículas de madeira leucena e resina poliuretana derivada de óleo de mamona
}

\author{
Particleboard manufactured with leucena wood and polyurethane resin castor oil based
}

\author{
Sergio Augusto Mello da Silva ${ }^{\mathrm{I}}$ André Luis Christoforo ${ }^{\mathrm{I}}{ }^{*}$ Túlio Hallak Panzera ${ }^{\mathrm{II}}$

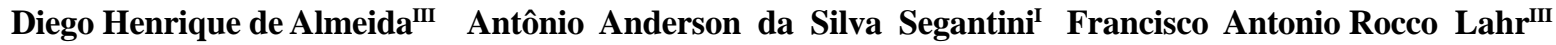

RESUMO

O presente trabalho objetivou avaliar as características físico mecânicas de chapas aglomeradas com partículas de leucena (Leucaena leucocephala) e resina poliuretana derivada de óleo de mamona, sendo investigados o módulo de resistência à flexão (MOR), adesão interna (AI), densidade aparente $\left(d_{a p}\right)$ e teor de umidade $\left(u_{m}\right)$. Os experimentos foram desenvolvidos com base nos procedimentos metodológicos da norma brasileira ABNT NBR 14810:2002. As chapas foram confeccionadas com pressão de compactação de $4 M P a$, temperatura de $90^{\circ} \mathrm{C}$, partículas com $5 \%$ de teor de umidade e adição de $10 \%$ de resina poliuretana monocomponente e bicomponente derivadas de óleo de mamona. Os resultados obtidos demonstraram que as densidades aparentes obtidas foram superiores às recomendadas pela norma brasileira, sendo os painéis confeccionados com resina bicomponente $22,67 \%$ superiores ao limite superior estabelecido pela normatização técnica brasileira. Os painéis confeccionados com resina poliuretana monocomponente apresentaram os maiores valores para o teor de umidade. O módulo de resistência à flexão dos painéis fabricados com resina bicomponente mostrou ser 43,7\% superior ao valor definido pela norma. A adesão interna dos painéis fabricados com resina monocomponente foi 2,5 vezes superior ao valor estipulado pela norma. Do intervalo de confiança entre médias para as resinas mono e bicomponente, apenas a adesão interna e densidade apresentaram equivalência estatística. Os coeficientes de determinação obtidos das regressões entre a adesão interna e densidade para ambas as resinas validaram o modelo de ajuste linear, sendo possível estimar a adesão interna com o conhecimento da densidade aparente das chapas.

Palavras-chave: resistência, flexão, adesão interna, teor de umidade.

\section{ABSTRACT}

This research aimed to test particleboard with leucena (Leucaena leucocephala) wood particles and polyurethane resin castor oil based. The response variables are: modulus of rupture $(M O R)$, internal adhesion (AI), apparent density $\left(d_{a p}\right)$ and wood moisture content $(u)$. The experiments were developed based on the methodological procedures of the ABNT NBR 14810:2002 standard. The particleboards were manufactured by hot-pressing at $4 \mathrm{MPa}$ and $90^{\circ} \mathrm{C}$, using timber particles with $5 \%$ of moisture content and $10 \%$ of monocomponent and bicomponent polyurethane resin. The higher moisture content was achieved when the monocomponent polyurethane resin was used. The bicomponent polyurethane resin provided a percent increase of $43.7 \%$ and $22.7 \%$ on the modulus of rupture and apparent density, respectively, when compared to the standard limit. The internal adhesion of the panels manufactured with monocomponent resin was 2.45 times higher than the standard limit. The confidence interval between means revealed that the internal adhesion and apparent density exhibited statistical equivalence. A good correlation between the internal adhesion and apparent density was found, for this reason it was possible to estimate the internal adhesion of the panels based on the apparent density data.

Key words: strength, bending, internal adhesion, moisture content.

\section{INTRODUÇÃO}

Várias pesquisas vêm sendo desenvolvidas na elaboração de chapas de partículas, com o objetivo de verificar a viabilidade da elaboração de painéis mediante certos fatores (pressão de compactação, temperatura de prensagem, proporção entre partículas e adesivos, tipos de adesivos etc.) e níveis experimentais estipulados (AKBULUT \& KOÇ, 2006; YEMELE et al., 2008), sendo os adesivos a base de ureia-formaldeído os mais utilizados (UYSAL, 2005).

Propriedades de resistências e rigidez de painéis confeccionados com fibras de madeiras de

'Departamento de Engenharia Civil, Universidade Estadual Paulista Júlio de Mesquita Filho (UNESP), Ilha Solteira SP, Brasil.

"Departamento de Engenharia Mecânica, Universidade Federal de São João del-Rei (UFSJ), Praça Frei Orlando, 170, Centro, 36307-352, São João del-Rei, MG, Brasil. E-mail: alchristoforo@yahoo.com.br. *Autor para correspondência.

II'Departamento de Engenharia de Estruturas, Escola de Engenharia de São Carlos (EESC), Universidade de São Paulo (USP), São Carlos, SP, Brasil. 
clones de álamo híbrido foram pesquisados por SHI et al. (2005). Os estudos revelaram que as fibras propiciaram produção de painéis com resistências mecânicas satisfatórias e com boa estabilidade dimensional.

O uso de espécies exóticas de madeira larch (Larix gmelinii e Larix sibirica) como matériaprima para a fabricação de painéis foi pesquisado por JUN LI et al. (2006), sendo investigados o módulo de elasticidade na flexão (MOE), módulo de ruptura à flexão (MOR) e adesão interna (AI). Os resultados obtidos para as propriedades mecânicas indicam ser possível o emprego de ambas as espécies de madeira na fabricação de painéis.

AKGÜLA \& ÇAMLIBELB (2008) estudaram a resistência e a rigidez de painéis de partículas fabricados com a madeira Rhododendron, tendo as partículas teor de umidade de $14 \%$ e unidas por adesivo a base de ureia-formaldeído. Os resultados obtidos das condições investigadas indicaram o emprego da madeira Rhododendron na fabricação de painéis.

A viabilidade de produção de painéis de partículas em clones de seringueira RRIM 2020 com quatro anos de idade foi estudada por SAFFIAN et al. (2011), sendo avaliados os módulos de elasticidade (MOE) e de ruptura (MOR). Os resultados encontrados indicaram ser possível a fabricação de painéis com os clones de seringueira avaliados.

Um aspecto a ser considerado na industrialização de painéis de partícula é a utilização de produtos que poluem o meio ambiente, principalmente por meio da emissão de gases. Nesse sentido é necessário o desenvolvimento de novos produtos, como é o caso do estudo proposto por BRADI et al. (2006), tendo avaliado a influência da mistura de óleo vegetal em matriz poliuretana na resistência mecânica de painéis de fibras de madeira. As análises realizadas possibilitaram concluir que é possível a utilização de misturas de óleo vegetal em matriz poliuretana na relação de 35:65 (em massa) para confecção de painéis de fibras de madeira.

A produção de chapas aglomeradas homogêneas com partículas de madeiras da Amazônia de baixa e média densidade (Erisma uncinatum, Nectranda lanceolata, Erisma sp.) foi estudada por SILVA \& LAHR (2007). As chapas foram confeccionadas com espessura nominal de $10 \mathrm{~mm}$, densidade nominal de $0,75 \mathrm{~g} \mathrm{~cm}^{-3}$, ao empregarem $10 \%$ de resina poliuretana bicomponente derivada de óleo de mamona e partículas com comprimentos entre 0,02 a $6 \mathrm{~mm}$, sob condições de prensagem, com temperatura de $90^{\circ} \mathrm{C}, 40 \mathrm{MPa}$ e tempo de $10 \mathrm{~min}$. Nas avaliações realizadas de acordo com a norma brasileira regulamentadora NBR14810 da Associação Brasileira de Normas Técnicas (ABNT), as chapas de partículas da madeira Nectranda lanceolata apresentaram os maiores valores de resistência, sendo estes superiores ao limite estabelecido pela norma citada.

DIAS et al. (2008) estudaram propriedades mecânicas de painéis de madeira aglomerada constituídos de resina poliuretana a base de mamona. Os resultados obtidos para o MOE não alcançaram o valor mínimo de $18 \mathrm{MPa}$, possivelmente justificado pela má distribuição do adesivo durante o processo de formação dos painéis.

Painéis de partículas aglomeradas com bagaço de cana e resina poliuretana derivada de óleo de mamona foram pesquisados no trabalho de FIORELLI et al. (2011), tendo investigado as propriedades: densidade, inchamento, absorção e módulo de elasticidade e de ruptura à flexão. Os resultados indicaram os materiais fabricados como sendo de alta densidade, recomendado para uso industrial, evidenciando a eficiência da resina poliuretana a base de mamona como adesivo.

PAES et al. (2011) estudaram o efeito da combinação da pressão $(2,0 ; 3,0 ; 3,5 \mathrm{MPa})$ e temperatura $\left(50 ; 60 ; 90^{\circ} \mathrm{C}\right)$ em painéis de partículas com resíduos de madeira de Pinus elliottii e resina poliuretana derivada de mamona nas variáveis resposta: densidade, inchamento e absorção de água (0-2h; 2-24h; 0-24h); módulo de elasticidade e de ruptura à flexão, arrancamento de parafuso e ligação interna, concluindo que as combinações $3,0 \mathrm{MPa}$ e $90^{\circ} \mathrm{C}$ e $3,5 \mathrm{MPa}$ e $60^{\circ} \mathrm{C}$ apresentaram os melhores resultados, comprovando ser a temperatura de prensagem a variável mais significativa quanto a qualidade das chapas elaboradas.

Painéis de madeira de reflorestamento e chapas de partículas de bagaço de cana-de-açúcar com resina poliuretana bicomponente derivada de óleo de mamona foram estudados por SARTORI et al. (2012), como solução alternativa para o sistema de fechamento lateral do tronco coletivo de centro de manejo para bovinos de corte. As propriedades físico-mecânicas obtidas comprovaram a eficiência do modelo estrutural proposto para uso em centro de manejo.

Segundo a Associação Brasileira da Indústria de Painéis de Madeira - ABIPA (2006), o Brasil é um dos países mais avançados do mundo na fabricação de painéis de partículas, com o maior número de fábricas de última geração, cuja produção anual equivale atualmente a $612.000 \mathrm{~m}^{3}$, representando, para produção mundial, um percentual 
muito baixo, considerando-se o potencial madeireiro do país e as tecnologias instaladas.

Considerando-se os aspetos da atual produção de painéis de partículas do Brasil e a necessidade de estudos que possibilitem a utilização de novos adesivos, este trabalho objetivou avaliar, com o auxílio do documento normativo brasileiro ABNT NBR 14810:2002, as propriedades mecânicas: módulo de resistência à flexão (MOR) e adesão interna (AI) e físicas: densidade aparente $\left(\mathrm{d}_{\mathrm{ap}}\right)$ e teor de umidade $\left(\mathrm{u}_{\mathrm{m}}\right)$ de painéis de partículas produzidos com resina poliuretana mono e bicomponente, derivadas de óleo de mamona e fibras de madeira de leucena (Leucaena leucocephala), espécie de madeira não explorada na elaboração de painéis, com o intuito de avaliar o potencial dos materiais fabricados.

A leucena (Leucaena leucocephala) é originária da América Central, espécie muito prolífera e de rápido crescimento, sendo disseminada nas Regiões Sul, Sudeste e Nordeste do Brasil. É de difícil erradicação, fazendo parte da lista das 100 espécies invasoras mais agressivas do planeta (COSTA, 1987).

Por causa da grande quantidade de árvores de leucena no município de Ilha Solteira e em decorrência da necessidade de se dar um destino para a madeira proveniente de podas, foi elaborado um projeto de pesquisa entre a Universidade Estadual Júlio Mesquita Filho (UNESP), Universidade de São Paulo (USP) e Casa da Agricultura de Ilha Solteira, com objetivo de pesquisar a produção de chapas com partículas de leucena (SILVA, 2008). $\mathrm{O}$ aproveitamento de resíduos de madeira em Ilha Solteira se constitui de um projeto do Parque Tecnológico, que visa a um consórcio entre a UNESP e empresas madeireiras da região Noroeste do Estado de São Paulo para produção de chapas aglomeradas de madeira por meio de incubadoras tecnológicas.

Além da caracterização dos painéis, procurou-se também investigar a relação entre a adesão interna e a densidade aparente, com o intuito de verificar a possibilidade de estimar a adesão interna com o conhecimento da densidade.

\section{MATERIAL E MÉTODOS}

Pequenas toras de madeira leucena, com 10 a $20 \mathrm{~cm}$ de diâmetro e $100 \mathrm{~cm}$ de comprimento foram selecionadas para serem submetidas ao processo de fragmentação para produção das partículas, com comprimento aproximado de $6 \mathrm{~mm}$.
As chapas foram confeccionadas em prensa hidráulica com controle de temperatura e pressão, sendo empregadas, segundo recomendações de SILVA \& LAHR (2007), pressão de compactação de $4 \mathrm{MPa}$, temperatura de $90^{\circ} \mathrm{C}$ e ciclo de prensagem de $3 \mathrm{~min}$ iniciais, abertura da prensa por $30 \mathrm{~s}$ e $7 \mathrm{~min}$ de prensagem final.

Foram confeccionadas duas chapas de partículas para cada tipo de resina, mono e bicomponente, nas dimensões de $400 \times 400 \times 10 \mathrm{~mm}$. Passadas 72 horas após a prensagem, as chapas foram esquadrejadas nas dimensões de $350 \times 350 \times 10 \mathrm{~mm}$.

Para avaliação do módulo de resistência à flexão (MOR), foram extraídos sete corpos de prova (CP) de cada chapa, elaborada com resina poliuretana monocomponente, e outros sete de cada chapa confeccionada com resina poliuretana bicomponente, nas dimensões nominais de $50 \times 250 \times 10 \mathrm{~mm}$, totalizando 14 amostras por condição.

Para avaliação da adesão interna, foram extraídas duas amostras de cada corpo de prova utilizado para estudo do MOR, nas dimensões $50 \times 50 \times 10 \mathrm{~mm}$, conduzindo a 28 amostras por tipo de resina, e para avaliação da densidade aparente e teor de umidade, foram extraídas dos painéis 28 amostras com dimensões de $50 \times 50 \times 10 \mathrm{~mm}$ para cada tipo de resina.

Com relação à adição das resinas, foram adotados procedimentos distintos em função das características de cada resina. Para o caso da monocomponente, utilizaram-se $10 \%$ de resina em função da massa seca das partículas, sendo, em seguida, adicionadas as partículas. No caso da resina bicomponente, consideraram-se para sua composição a relação de uma parte de poliol para uma de pré-polímero, totalizando $10 \%$ de resina em função da massa seca das partículas. A adição da resina nas partículas ocorreu considerandose primeiramente a introdução do poliol e, após homogeneização do poliol às partículas, a adição do pré-polímero.

O intervalo de confiança entre médias, com nível de confiabilidade de $95 \%$, foi utilizado para verificar a equivalência estatística entre o emprego das resinas poliuretanas mono e bicomponente nas variáveis-resposta investigadas, expresso pela Equação 1, sendo $\mu$ a média populacional das diferenças, $\bar{x}_{\mathrm{m}}$ a média aritmética amostral das diferenças, $n$ o tamanho da amostra, $S_{m}$ o desvio padrão amostral das diferenças e $\mathrm{t}_{\alpha / 2, \mathrm{n}-1} \mathrm{o}$ valor tabelado pela distribuição " $\mathrm{t}$ " de Student, com $n-1$ graus de liberdade e nível de significância $\alpha$. 


$$
\bar{x}_{m}-t_{\alpha / 2, n-1} \cdot S_{m} / \sqrt{n} \leq \mu \leq \bar{x}_{m}+t_{\alpha / 2, n-1} \cdot S_{m} / \sqrt{n}
$$

O teste de Anderson-Darling foi utilizado na verificação de distribuição normal para os conjuntos dos valores das variáveis investigadas, validando o emprego do intervalo de confiança.

\section{RESULTADOS E DISCUSSÃO}

Os resultados obtidos para as propriedades físicas e mecânicas das chapas são apresentados na tabela 1. Segundo a norma brasileira ABNT NBR 14810:2002, o valor estipulado para o módulo de resistência à flexão (MOR) deve ser de no mínimo $18 \mathrm{MPa}$, devendo ser de 0,40MPa o valor mínimo para a adesão interna (AI).

Os P-valores do Teste de normalidade de Anderson-Darling para as propriedades mecânicas variaram entre 0,473 a 0,691 , sendo todos superiores a 0,05, comprovando ser normal a distribuição dos dados encontrados (MONTGOMERY, 2005), validando o emprego dos intervalos de confiança calculados.

De acordo com os resultados do MOR e AI apresentados na tabela 1, constata-se que as chapas confeccionadas com as resinas poliuretanas monocomponente e bicomponente apresentaram valores superiores aos valores limites definidos pela norma NBR 14810:2002, sendo a adesão interna obtida com o uso de ambas as resinas, duas vezes superior ao limite mínimo estipulado.

Com relação a propriedades físicas, os intervalos dos valores para a densidade aparente $(\mathrm{g}$ $\mathrm{cm}^{-3}$ ) e teor de umidade (\%) estipulados por norma são $[0,55 ; 0,75]$ e $[5 ; 11]$, respectivamente.

Os P-valores do teste de normalidade de Anderson-Darling para as propriedades físicas variaram entre 0,536 a 0,622 , sendo todos superiores a 0,05 , comprovando ser normal a distribuição dos dados encontrados.

Conforme se observa na tabela 1 , das propriedades físicas investigadas, a densidade não atendeu aos requisitos da norma $\mathrm{ABNT}$ NBR 14810:2002, tendo as resinas mono e bicomponente valores de $17 \%$ e $22,67 \%$ superiores, quando comparados com o limite superior estipulado pela norma. Com relação ao teor de umidade, os painéis confeccionados com ambas as resinas atenderam aos requisitos da norma, sendo os maiores teores de umidade obtidos como uso da resina poliuretana monocomponente.

$\mathrm{Na}$ tabela 2 constam os resultados dos intervalos de confiança $(\mu)$, graus de liberdade (DF) e os respectivos P-valores das variáveis-resposta, sendo confrontadas pelo tipo de resina utilizada na confecção dos painéis.

Os resultados do intervalo de confiança para a adesão interna e densidade aparente demonstram equivalência estatística, não havendo diferenças significativas entre o emprego de ambas as resinas na obtenção dessas propriedades, o mesmo caso não ocorrendo com o módulo de resistência à flexão e teor de umidade.

Os valores médios dos módulos de resistência à flexão para os materiais fabricados com as

Tabela 1 - Propriedades físicas e mecânicas obtidas dos painéis fabricados.

\begin{tabular}{|c|c|c|c|c|}
\hline \multirow{2}{*}{ Propriedades mecânicas } & \multicolumn{2}{|c|}{-------Resina poliuretana monocomponente------ } & \multicolumn{2}{|c|}{------Resina poliuretana bicomponente------ } \\
\hline & MOR (MPa) & $\mathrm{AI}(\mathrm{MPa})$ & MOR (MPa) & $\mathrm{AI}(\mathrm{MPa})$ \\
\hline Média & 19,37 & 1,38 & 25,87 & 1,26 \\
\hline Desvio padrão & 3,02 & 0,25 & 3,97 & 0,33 \\
\hline Coef. de variação & 0,16 & 0,18 & 0,15 & 0,27 \\
\hline Máximo & 25,35 & 1,93 & 31,88 & 2,10 \\
\hline Mínimo & 14,11 & 0,86 & 18,32 & 0,77 \\
\hline \multirow{2}{*}{ Propriedades físicas } & \multicolumn{2}{|c|}{------Resina Poliuretana Monocomponente------ } & \multicolumn{2}{|c|}{------Resina Poliuretana Bicomponente----- } \\
\hline & $\mathrm{d}_{\mathrm{ap}}\left(\mathrm{g} \mathrm{cm}^{-3}\right)$ & $\mathrm{u}_{\mathrm{m}}(\%)$ & $\mathrm{d}_{\mathrm{ap}}\left(\mathrm{g} \mathrm{cm}^{-3}\right)$ & $\mathrm{u}_{\mathrm{m}}(\%)$ \\
\hline Média & 0,88 & 10,11 & 0,92 & 8,00 \\
\hline Desvio Padrão & 0,04 & 0,51 & 0,05 & 0,41 \\
\hline Coef. de variação & 0,04 & 0,05 & 0,05 & 0,05 \\
\hline Máximo & 0,93 & 10,80 & 0,99 & 8,70 \\
\hline Mínimo & 0,82 & 9,10 & 0,82 & 7,50 \\
\hline
\end{tabular}

Ciência Rural, v.43, n.8, ago, 2013. 
Tabela 2 - Resultados do intervalo do confiança das variáveis investigadas.

\begin{tabular}{lllll}
\hline Variável resposta & P-valor & GL & Intervalo de confiança & Condição \\
\hline MOR & 0,003 & 28 & $-21,65=\mu=-10,38$ & Não equivalente \\
$\mathrm{AI}$ & 0,191 & 49 & $-1,55=\mu=1,61$ & Equivalente \\
$\mathrm{d}_{\mathrm{ap}}$ & 0,328 & 53 & $-0,22=\mu=0,15$ & Equivalente \\
$\mathrm{u}_{\mathrm{m}}$ & 0,000 & 52 & $0,01=\mu=3,86$ & Não equivalente \\
\hline
\end{tabular}

resinas mono e bicomponente foram, respectivamente, 7,60 e 43,7\% superiores aos estipulados pela norma, sendo o MOR oriundo dos painéis fabricados com resina poliuretana bicomponente $33,56 \%$ superior ao MOR dos painéis confeccionados com a resina monocomponente.

Com o intuito de relacionar a adesão interna com a densidade aparente para ambas as resinas, foram realizadas duas regressões lineares distintas, uma para cada tipo de resina. As equações de regressão entre essas variáveis para as resinas poliuretanas mono e bicomponente ajustadas foram $\mathrm{AI}=-7,11+9,69 \cdot \mathrm{d}_{\text {ap }} \mathrm{e}$ $\mathrm{AI}=-5,75+7,59 \cdot \mathrm{d}$, respectivamente, considerando um nível de significância igual a $0,05, \operatorname{com~R}^{2}$ (Adj) de 0,94 e 0,91 , P-valores iguais a 0,000 e 0000 e valores F de significação de 0,01 e 0,00 , comprovando serem válidas e significativas as relações lineares obtidas entre as variáveis.

\section{CONCLUSÃO}

O emprego de $10 \%$ de resina poliuretana monocomponente e bicomponente, juntamente com os demais fatores investigados na elaboração dos painéis, não atendeu de forma satisfatória apenas a densidade aparente, sendo os maiores valores encontrados nos painéis fabricados com a resina poliuretana bicomponente.

Os valores do MOR e AI foram superiores aos estipulados pela norma brasileira, apresentando ser a adesão interna dos painéis fabricados com resina monocomponente 2,45 vezes superior ao valor limite estipulado.

Os coeficientes de determinação das regressões entre a adesão interna e densidade aparente para ambas as resinas comprovaram a linearidade entre as variáveis, possibilitando-se estimar a adesão interna dos painéis com o conhecimento das suas respectivas densidades.

Mesmo considerando os resultados significativos, principalmente para as propriedades mecânicas, faz-se necessário ajustar as variáveis do processo de confecção das chapas, buscandose o emprego de quantidades menores de resina, objetivando diminuir os custos finais das chapas.

\section{REFERÊNCIAS}

AKBULUT, T.; KOÇ, E. The effect of the wood species on the roughness of the surface and profiled areas of medium density fiberboard. Wood Research, v.51, n.2, p.77-86, 2006.

AKGÜLA, M.; ÇAMLIBELB, O. Manufacture of medium density fiberboard (MDF) panels from rhododendron (R. ponticum L.) biomass. Building and Environment, Part special: Building Performance Simulation, v.43, n.4, p.438-443, 2008.

ASSOCIAÇÃO BRASILEIRA DA INDÚSUTRIAS DE PAINÉIS DE MADEIRA (ABIPA) - PRODUTOS E TECNOLOGIAS. Sobre consumo mundial de aglomerado em 2004/2005. São Paulo (SP), 2006. Disponível em: 〈htpp://www.abipa.org.br〉. Acesso em: 28/06/2011.

ASSOCIAÇÃO BRASILEIRA DE NORMAS TÉCNICAS (ABNT). Chapas de madeira aglomerada. Rio de Janeiro, 2002. ABNT NBR 14810-1, 2, 3.

BRADI, K.H. et al. Effect of filler-to-matrix blending ratio on the mechanical strength of palm-based. Polymer International, v.55, n.2, p.190-195, 2006.

COSTA, N. L. Recomendações técnicas para o cultivo de leucina. Porto Velho: EMBRAPA-UEPAE, 1987. 8p. (Comunicado técnico 50).

DIAS, F. M. Aplicação de resina poliuretana à base de mamona na fabricação de painéis de madeira aglomerada. In: LAHR, F. A. R. Produtos derivados da madeira. São Carlos: EESC/USP, 2008. p. 37-160.

FIORELLI, J. et al. Painéis de partículas à base de bagaço de cana e resina de mamona - produção e propriedades. Acta Scientiarum Technology, v.33, n.4, p.401-406, 2011.

JUN LI, S. et al. Feasibility of using two exotic larch species as raw material for medium density fiber board panel manufacturing. Forest Products Journal, v.56, n.5, p.48-52, 2006.

MONTGOMERY, D.C. Design and analysis of experiments. 6 . ed. Arizona: John Wiley \& Sons, 2005, 521 p.

PAES, J. B. et al. Qualidade de chapas de partículas de pinus elliottii coladas com resina poliuretana sob diferentes combinações de pressão e temperatura. Ciência Florestal, v.21, n.3, p.551-558, 2011. 
SAFFIAN, H. A. et al. Feasibility of manufacturing a medium density fibreboard made of 4-year old rubber tree RRIM 2020 clone. Key Engineering Materials - Composite Science and Technology, v.471, p.839-844, 2011.

SARTORI, D.L. et al. Painel em madeira de reflorestamento e chapas de partículas para instalações rurais. Floresta e Ambiente, v. 19, n. 2 , p. $171-178,2012$.

SHI, J. L. et al. Flexural properties, internal bond strength and dimensional stability of medium density fiberboard panels made from hybrid poplar clones. Wood and Fiber Science, v37, n.4, p.629-637, 2005.

SILVA, S. A. M. Chapas de partículas confeccionadas com resíduos de madeiras tropicais de baixa densidade. 2008, 182p. Relatório Técnico-Científico (Pós-Doutorado em Engenharia de Estruturas) - Universidade de São Paulo, Escola de Engenharia de São Carlos, Departamento de Engenharia de Estruturas, São Carlos-SP.

SILVA, S.; ROCCO LAHR, F. A. Chapas de partículas confeccionadas com resíduos de madeiras tropicais de baixa densidade. In: Francisco Antonio Rocco Lahr. Reciclagem de resíduos para a construção civil. Belo Horizonte: FUMEC, 2007. Cap.14, p.343-365.

UYSAL, B. Withdrawal strength of various laminated veneer dowels from composite materials. Wood and Fiber Science, v.37, n.2, p.213-219, 2005.

YEMELE, M. C. N. et al. Effects of bark content and particle geometry on the physical and mechanical properties of particleboard made from black spruce and trembling aspen bark. Forest Products Journal, v.58, n.11, p.48-56, 2008. 